\title{
Understanding and addressing the stigma of mental illness with ethnic minority communities
}

\author{
Lee Knifton \\ School of Applied Social Science, University of Strathclyde, Glasgow, Scotland, UK
}

AвsтRACT: Higher income societies have moved from institutional to community-based care for people experiencing mental illness. However, stigma and discrimination persists and undermines help-seeking, recovery and life chances. Mental illness prevalence is higher amongst communities that face multiple prejudices and disadvantages within society, including black and minority ethnic communities who may experience migration trauma, racism, acculturation and adverse social circumstances. This study examines beliefs, stigma and the effectiveness of existing national mental health campaigns with Pakistani, Indian and Chinese heritage communities in Scotland, UK, using community based participatory research. Community organisers were trained and supported to co-facilitate focus groups with 87 people using a range of languages. Whilst diversity within and between communities was apparent, important trends emerged. People with mental illness experience high levels of stigma from communities. Families experience significant associated stigma. This shame combines with culturally inappropriate services to reduce help seeking from mental health services, friends and families. Existing anti-stigma campaigns have failed to reach or engage with communities due to a combination of practical issues such as the use of inappropriate language, imagery and media, but also due to assuming western medical concepts of illness. Participants suggested a new model for national campaigns placing greater emphasis upon community development, cultural events, positive contact and dialogue with families, faith leaders and youth groups. National anti-stigma programmes must develop more effective partnerships with communities or risk magnifying existing inequalities. Despite a range of national programmes to address stigma towards people with mental illness, it remains as the major barrier to social inclusion for people with mental illness living in communities. Stigma is particularly severe if people have a mental illness and are also economically disadvantaged or face multiple stigmas. By adopting Community Based Participatory Research (CBPR) approaches with three black and ethnic minority communities in Scotland, this study makes the case that stigma can only be understood and effectively addressed with reference to its socio-cultural context.

KeYwords: mental illness, sociology, ethnic minority, stigma, discrimination, culture

$\mathrm{T}$ he way in which we support people with enduring mental health problems in affluent countries is changing. Deinstitutionalisation and recovery-focused models of care are frequently accompanied by rights-based legislation in relation to healthcare treatment, employment and education. Yet in contemporary societies, the intense stigma and discrimination that people with mental health problems continue to face results in a series of 'social injuries' that are profoundly damaging (Rogers \& Pilgrim, 2003).

Sociologists Link and Phelan (2001) provide a strong model for understanding mental health stigma. They acknowledge that stigma includes a combination of inaccurate or distorted beliefs, negative attitudes and discriminatory behaviour. They describe stigma as the co-occurrence of labelling of difference, stereotyping, separation of 'us' and 'them', followed by discrimination and status loss. This model acknowledges that it is easier to discriminate against groups who have less power, and that stigma can exist at a number of levels in society: Internalised stigma (or anticipated discrimination) for the person affected; social stigma experienced in everyday interactions with friends, family and the wider community; and structural stigma (or institutional discrimination) whereby prejudice is embedded into legal systems, the media, cultural and businesses institutions. Family and friends can also be stigmatised by association with the labelled person or group although this dimension has received less attention (Goffman, 1963).

The impact of stigma is profound. People with mental health problems frequently delay help-seeking for fear of the social consequences (Schomerus \& Angermeyer, 2008). Life chances 
and opportunities are consistently restricted (Rosenfield, 1997). Harassment in the community remains a common phenomenon (Berzins, Petch, \& Atkinson, 2003). Employment rates remain at under 20\% across Europe compared to over $40 \%$ of all people with a disability (Williams, Copestake, Eversley, \& Stafford, 2008) and stigma from employers and colleagues is a major cause (Arthur, Knifton, Park, \& Doherty, 2008; Faulkner \& Layzell, 2000). The cumulative effects of this income and status loss, combined with fear of rejection from friends, families and partners, frequently results in lowered self-esteem (Wright, Gronfein, \& Owens, 2000). Enduring stigma can lead to people living in states of constant stress, worsening the initial mental health problem (Link \& Phelan, 2006). Under-investment in health services from governmental institutions contributes towards a gap between mental health need, services and support, especially amongst disadvantaged social groups (Patel et al., 2010).

In Scotland, the location of this study, stigma and discrimination against people with mental illness continues to be a major issue. Dangerousness remains a commonly reported belief in public surveys and in the media, along with notions that people are to blame for their condition, are unwilling or unable to work, make little social contribution and are unsuitable as partners (Knifton \& Quinn, 2008). Beliefs about danger increased as we moved from institutions to community care, with a shift from 'becoming mad' to 'fear of the other' (Maclean, 1969). Danger and unpredictability remain highly associated with people with severe and enduring mental illnesses including psychoses and schizophrenia. Other conditions, such as depression, attract more subtle forms of stigma related to ability to work and being difficult to talk to (Crisp, Gelder, Rix, Meltzer, \& Rowlands, 2000).

This study engages with communities that experience multiple forms of disadvantage and disempowerment, and the case for understanding and addressing mental health stigma in the context of inequalities is compelling. Globally the prevalence of mental health problems is high and increasing (World Health Organization, 2001) and the burden does not fall equally within societies. Those experiencing material and social disadvantage have much higher rates of mental illness (Pickett, James, \& Wilkinson, 2006). In particular mental illness and distress is caused and perpetuated by relative inequality, meaning that it is not the absolute levels of poverty or disadvantage that matter as much as the differences within a society between those who have most and those who have least. The greater the disparity, the greater the levels of mental illness and distress (Friedli, 2009). This social gradient in health was outlined in the UK by Marmot (2010) in his recent review and he recommended proportionate universalism as a response from health and public agencies to reduce health inequalities. This means that universal health and social programmes should invest more resources and effort into areas and communities according to the level of need.

In relation to mental health stigma specifically, greater prevalence is reported from members of communities in disadvantaged areas (Braunholtz, Davidson, Myant, \& O'Connor, 2006). So not only is someone more likely to experience mental illness in an area of deprivation, they may have less access to support, have fewer opportunities for employment, and will likely face a more stigmatising community response.

When people face multiple disadvantages, mental health stigma needs to be considered in this wider context. This study explores mental health stigma with the three largest black and minority ethnic (BME) communities in Scotland, comprising citizens with Pakistani, Chinese and Indian heritage who encompass a variety of cultures and religious beliefs. Mental health services and campaigns rarely target BME communities consistently. Yet BME communities frequently experience high rates of mental health problems linked to social circumstances such as unemployment, stress and living conditions (Williams \& Hunt, 1997) and life events such as family absence, acculturation and migration trauma (Myers, McCollam, \& Woodhouse, 2005). 
These problems are consistently exacerbated by racism from communities and services (Chakraborty, McKenzie, Hajat, \& Stansfeld, 2010). In the UK, BME communities frequently receive inadequate mental health service provision. In some cases, particularly amongst Asian minorities, services fail to reach or engage with communities where there is need. In other cases there can be an excess of compulsory admissions and restrictive treatments (Fernando, 2003). This can be attributed to racial prejudice and fear amongst practitioners. It is also seen as a consequence of prejudice embedded within psychiatry and the diagnostic process itself, which takes little account of cultural differences. So if mental health services are seen as part of a system that diminishes your cultural beliefs, or exerts restrictive power over you, then mental illness itself can become something to fear, avoid or reject. When that same system dominates campaigns to reduce stigma both the messages and their source may lack credibility. In Scotland this dual stigma shows little improvement despite various equalities initiatives (Grant \& Jackson, 2005). High levels of social isolation and significant racism are reported by all three major BME communities and dissatisfaction with services is highest amongst Chinese minorities (Heim \& MacAskil, 2006).

Layered onto these social issues, it is important to consider cultural beliefs as a potentially important factor influencing social stigma towards people with mental illness. In international cross-cultural studies the emerging trends are that the impact of stigma and discrimination affects similar domains of individual's lives, for example employment, community harassment or marriage. However the intensity of impact varies and the forms and nature of stigmatising beliefs that people and communities hold also varies widely according to cultural beliefs (Van Brakel, 2006).

There is a significant amount of literature on cross-cultural dimensions of mental illness and social stigma. Fabrega (1991) reviewed the literature on psychiatric stigma in India, China and in Islamic countries including Pakistan, the countries of origin of the minority communities in this study, and found consistently high levels of stigma. More recent reviews across several Asian countries by Ng (1997) and Lauber and Rossler (2007) highlight variations within and across countries and cultures in the intensity of stigma. However overall stigma and discrimination are prevalent and common patterns emerge. Dangerousness is a common belief and often leads to social distance and isolation. Families can both stigmatise the person with mental illness and in turn, themselves be stigmatised through association by the community. A consequence is that relatives are devalued as potential marriage partners.

It is also important to acknowledge that psychiatric illness categories are western cultural constructs. Other cultures may not conceptualise distress as illness (Kleinman, 1987). Many Asian cultures use supernatural and religious explanations for what psychiatry sees as severe mental illnesses and common mental health problems are frequently expressed as physical health problems, which can be less socially disadvantageous (Fabrega, 1992; Lauber \& Rossler, 2007). Knowing who is labelled as having a mental illness in a community is fundamental to knowing how to approach tackling stigma.

We are not certain how beliefs and stigmas that are common in people's countries of origin change amongst first, second and third generation minorities. There is a lack of research concerning the nature and impact of mental health stigma amongst BME communities in the UK or Scotland specifically. National mental health surveys in Scotland on awareness and attitudes did not focus upon BME communities. Of the UK studies available, family reputation and marriage concerns do feature heavily amongst South Asian minority communities. There is evidence that some cultural and religious beliefs about causation can lead to shame and blame (Hatfield, Mohammad, Ahim, \& Tanweer, 1996; Tabassum, Macaskill, \& Ahmad, 2000). Shame, guilt and losing 'face' also emerged in studies with Chinese ethnic minority communities. Perceptions of dangerousness are reported along with hiding the 
condition from friends, family and health services (Blackwell, 1997; Li, Logan, Yee, \& Ng, 1999; Wong \& Richman, 2004; Yeung, 2004).

Wider international studies with BME communities in affluent countries indicate that culture does play a significant role in shaping mental health stigma. For example in the US, Gary (2005) explores the mental health stigma experienced by four ethnic minority communities. She notes how the prejudice experienced by ethnic group affiliation leads to dual stigma, and results in reduced help-seeking and a widening of mental health disparities.

Scotland has had a national programme to improve the public's mental health since 2003. This has developed as a public health approach and aims to promote positive mental health for all, prevent common disorders, and enhance the quality of life of people with mental illness. This includes a national campaign to reduce the stigma associated with mental illness, now in its eighth year. The campaign is well funded in international terms and to date activities of the campaign have been aimed at the general population or specific setting such as workplaces or schools. The main methods that it uses to tackle stigma include national advertising campaigns with key messages about stigma, media lobbying to protest against negative reporting, supporting local mental health groups to develop antistigma events and persuading major organisations to pledge to reduce stigma associated with mental illness. There is a focus upon producing resources such as posters and flyers for general distribution by mental health groups, health providers and government agencies. It describes itself as adopting a social marketing approach. Yet there is compelling evidence that mental illness stigma must be understood, and perhaps addressed, in relation to its social and cultural context. Little research has been undertaken with BME communities in Scotland to understand if there are important differences in beliefs about mental illness or patterns of stigma, or the extent to which the national programme has reached and influenced these communities.
This study undertakes research with members of the three largest BME communities in Scotland that have migrated from China, Pakistan and India. We explored commonly held beliefs about mental illness, patterns of stigma and discrimination and perceptions about the current national anti-stigma campaign in order to inform future action.

\section{Methods}

A broad based community coalition was established over the 2-year duration of the study, involving 12 partner organisations including universities, mental health agencies, the national anti-stigma campaign team and community groups representing the three largest BME communities in Scotland. This was an example of a community of learning and practice (Wenger, 1998). This coalition supported the study through a series of meetings with researchers helping to identify community projects and advising on methods of engagement. By developing a research partnership of stakeholders who both affect and are affected by mental illness and stigma the research was intended to be more equitable and collaborative (Cargo \& Mercer, 2008). Drawing upon different strengths and insights can lead to changes that improve health (Israel, Schulz, Parker, \& Becker, 1998). This coalition also has the potential to move from learning to action and use the research findings to help to develop improved methods of tackling stigma with BME communities (Israel et al., 1998).

In previous health research the coalition indicated that many people in BME communities felt like research subjects rather than partners. Staff in the community projects were keen to develop capacity in research skills, but also sought assurances that they would be involved in any future projects to improve mental health. This study, therefore, adopted a CBPR approach. This approach is based upon the belief that research is strengthened when formal researchers from outside the community partner with community representatives and organisers who have direct inside knowledge, experience and access to the communities 
(Minkler, 2004). Community organisers were drawn from the Chinese, Pakistani and Indian ethnic minority communities and shared their inside knowledge of community structures and responses to mental health issues in order to shape the design and focus of the study. In return the researchers helped to develop the formal research capacities of the community partners through training sessions and mentoring. They jointly developed the research method and materials, identified participants and collected data. CBPR was seen as particularly relevant in this context as it builds upon community strengths, can create more trust about a health topic that is heavily stigmatised, and sees people in the context of their social and cultural environments (Minkler \& Wallerstein, 2003).

The study aimed to gain an in depth understanding of mental illness and stigma in its social and cultural context and its meanings to participants. Qualitative research was adopted to enable us to capture subjective experiences and complexity. Focus groups were identified as the most culturally appropriate method across the communities involved. They also provided for a safe and supportive setting. Focus groups allow participants to share their collective knowledge more fully, and importantly why these views are held (Bryman, 2004). They enable participants to raise issues and explore and contextualise them, and the researchers to reflect back to participants to ensure shared understandings. The focus group schedule was developed in conjunction with community agencies and covered: Beliefs about mental illness and its causes; stigma and discrimination in communities; and opinions about the national anti-stigma campaign. Groups lasted between 90 minutes and 2 hours. Questions about mental health, stigma and discrimination were asked in the third person 'in your community' rather than about the person or people themselves. It was felt that this would elicit more honest findings and minimise social desirability bias.

The community projects were responsible for recruiting to and hosting the focus groups.
Ten focus groups were conducted involving 87 participants. The sampling was designed to include a mix of participants according to age, gender and faith. Within this outline framework, participants were recruited through community organisations using purposive sampling, and through local advertising by projects. This means that it was not important to be 'representative' but rather typical. Urdu, Punjabi, Hindi and Cantonese and translators were provided in the relevant focus groups to ensure that we could reach and engage with participants who did not have good English language skills.

The focus groups were co-facilitated by experienced formal researchers alongside community workers from participating BME community projects who were trained in research methods. To ensure that complex and subtle points were not missed or misinterpreted, facilitators regularly checked emerging points and themes with the group throughout the session. The facilitators and translators also jointly reviewed the focus group after the session to clarify any points that were specific to certain cultures or faiths, or which were difficult to translate. The focus group data was analysed systematically to allow key themes and issues to be identified. Data was analysed using our initial 'focusing factors' (Silverman, 2004) of 'beliefs about mental illness', 'stigma' and 'a national anti-stigma campaign'. A grounded theory technique was used to analyse the data for emerging themes and topics. Comments were analysed, described and labelled and then grouped into broad categories. Relationships between categories were examined within and between the groups and key themes identified. Whilst the analysis followed the principles of Glaser and Strauss' (1967) Grounded Theory, in practice CBPR involves researchers sharing concepts, perspectives and theoretical positions in designing the study and analysing the data. Therefore the process inevitably involved 'subtle interplay between theory, concepts and data' (Silverman, 2004) and could not be entirely inductive (see Corbin \& Strauss, 2008). 


\section{Findings}

The findings across the groups were both rich and complex. There was general agreement that mental health stigma and its consequences were profound and deeply embedded across and within the three communities. There was considerable variation within and between communities about perceived causes and meanings associated with mental illness and stigma. Older and lower income participants and first generation citizens held more traditional beliefs. Interestingly older and younger generations were well aware of the beliefs of each other. For the purposes of this paper, we focus upon beliefs and stigma and then the more specific data that relates the provision of mental health services and anti-stigma campaigns. The main themes are outlined in four sections: Beliefs and stigma; mental health treatment and services; the existing national anti-stigma campaign; and alternative models and approaches to engaging with BME communities.

\section{Beliefs and stigma associated with mental health problems}

Across the ethnic minority groups, high levels of mental health problems were attributed to a combination of stressful social circumstances faced by many people including economic deprivation, greater social isolation and pressures of family responsibilities. Amongst Chinese minority communities this was consistently reported as pressures of life. Newer migrants highlighted how processes of migration lead to particular stresses including often living in poor accommodation, language barriers and consistently experiencing high levels of racism from the wider community. South Asian participants reported poor living conditions, prejudice and violence more directly as contributing to mental health problems.

Mental health problems were seen to be a prevalent across the communities, however respondents mainly referred to more severe and enduring mental health problems rather than more common conditions such as anxiety and depression. Most participants resisted the idea that the latter are medical conditions as they are more commonly seen as responses to social problems, crises of faith or just 'part of life'. Amongst the more 'severe conditions' many people in communities frequently do not differentiate conditions such as schizophrenia or bipolar illness and more commonly view them as 'just madness'.

In addition to mental health problems being a major issue, all groups reported that people with mental health problems are frequently and heavily stigmatised within communities. This stigma takes several forms and often relates to underlying cultural and religious beliefs about causes. One set of explanations framed mental health problems in the context of blame for example as a punishment from God for a sin in a person's current or past life, or caused by spirits or jinn. Each of the Chinese and Hindu groups discussed karma linked to blame. A further theme was communities viewing mental illness as inherited through families. This frequently taints not just the person affected but the whole family within the community. This was reported across communities but the strongest examples emerged amongst Hindu, Sikh and Muslim communities. Here, respondents described examples of how extended family structures and a focus upon marriage magnifies the impact of stigma upon the person and the whole family. To maintain family reputation could lead to hiding the problem and isolation for people affected.

Amongst a wide range of responses, a number of consistent beliefs were reported both across and within groups. These include people with mental health problems being dangerous and unpredictable, less intelligent, unable to work effectively, and unsuitable as a marriage partner. These are fundamental aspects of affected people's social identity that are diminished. They are compounded by commonly reported beliefs that severe mental illness is incurable, with little possibility for recovery. It is therefore unsurprising that across all community groups 'shame' emerged as the most common response to mental health problems. This was expressed in different ways such as 'face' amongst Chinese communities, but with similar consequences.

Many of these findings about beliefs and stigma have been identified in previous 
international cross-cultural studies outlined in the introduction. Kleinman (1987), Fabrega (1992) and Lauber and Rossler (2007) consistently demonstrate that concepts of mental health may not be culturally constructed as illness, that stigma can affect families and not just individuals, and that beliefs about danger and unsuitability for marriage can lead to shame or social isolation for the person affected. Our findings highlight how these beliefs persist amongst migrant communities in Scotland. They reinforce some of the English studies with first and second generation Asian ethnic minority communities, which found that religious and cultural beliefs still lead to shame and blame (Hatfield et al., 1996; Tabassum et al., 2000). The study does not enable us to make detailed comparisons within and between communities. However, it was reported that more traditional beliefs were held less strongly by younger generations, professionals and those with higher economic status.

\section{Perceptions of mental health treatment and services}

Groups explored both the experience of mental health services and reasons for or against seeking treatment. Common themes about treatment emerged from each of the BME community discussions.

Stigma and recovery pessimism reduce helpseeking: One reason for avoiding help-seeking from health services stated by all generations was the shame associated with mental illness amongst communities, tying in with international research (Fabrega, 1991). This shame was also experienced by families within communities, which reduced support and motivation for individuals to seek medical help. Amongst older and first generations recovery-pessimism was common. A consistent reason for not seeking help from mental health services was a belief that it would not be effective in improving the mental health condition, that 'It will not work' or 'there is no cure'.

Many participants from Muslim, Hindu and Sikh heritage talked about a community belief that mental health problems are not an illness - but are caused by factors such as the will of
God, inheritance, 'black magic' or 'spirits'; consequently medication is not seen to help. Many community members were reported as holding a number of beliefs about causation in parallel; including social, religious and medical explanations which, again, supports wider international findings on cultural beliefs and stigma (Lauber \& Rossler, 2007). For a high proportion of respondents, religious leaders were seen as the first and foremost point of contact and support, particularly for those with Muslim, Hindu and Sikh heritage. Traditional treatments were reported as a common alternative to medical help seeking amongst those who have migrated from mainland China and Hong Kong. Social responses to treating mental illness permeated group discussions across communities, for example the belief that with family support people 'will get over it' or that 'Lots of love will solve it'.

Confidence with language was an important factor in using mental health services. Newer and first generation migrants, and those with lower confidence in English language ability will frequently seek help from religious leaders, as respected members of their community with whom they can communicate easily, rather than seeking help outside the community. Although interpreters are used across the three communities in other areas of life, there were perceived issues of confidentiality and a sense that it is difficult to convey feelings and emotions accurately through an interpreter.

The groups did not cite overt prejudice from health services as a reason for avoiding mental health services. However, respondents explained that there was a level of general distrust of social workers and doctors in some sections of the communities. Participants expressed more direct fear of being asked 'too many questions' either by the doctor or by others in the waiting room. They also expressed a lack of trust in personal information remaining confidential. A smaller proportion of respondents expressed fears of extreme measures. These concerns were expressed most strongly amongst respondents from Muslim communities, several of whom expressed concerns about 'ending up in an asylum'. Few people felt that provision of mental health services within and by 
members of the communities would significantly increase uptake, instead people generally restated concerns about confidentiality within relatively small communities.

Across the three BME communities, there was unanimity that members of the communities that were second and third generations, and those with high levels of education and language confidence were more likely to engage earlier and more readily with mental health services if needed. However, personal and family stigma was consistent across generations, acting as a barrier.

\section{Effectiveness of an existing national anti- stigma campaign}

Scotland has had a national anti-stigma campaign since 2003. Although there has been a sustained national campaign for several years, the groups expressed profound concerns about its reach, materials and overall approach in terms of engaging the BME communities. The findings were consistent across groups about its weaknesses, highlighting practical and conceptual issues.

A set of problems relate to language. The printed and advertising materials were all in English language and unavailable as translated resources. This excludes a significant number of people within the BME communities who have less confidence with English language from the campaign. This very likely includes those in most need. The language that is used is also quite subtle, cryptic and indirect and seen to require a good degree of proficiency to have a full effect. This criticism of language even applied to the term 'see me' which several of the groups felt was a particularly individualistic term and might have greater impact if it was a term that stressed people in the context of community and family.

Imagery was also raised. One concern across groups was the lack of multi-racial images. Images on materials were of white people. The groups felt that this should be more balanced, but not have such a high proportion of people from BME communities that it could imply these communities were 'mad' and risk reinforcing racism. Several of the groups also noted that they had not seen anyone from an ethnic minority community present in any of the television and media adverts, nor as volunteer speakers in the media. In addition to people, there were comments that the 'cultural activities' shown such as football were less likely to engage these three communities.

Members of all groups felt that national campaign materials had not reached their communities. They were distributed primarily through health services and local councils, but also through mental health consumer groups. Few of the campaign materials had been seen in religious, cultural or community centres. Existing press, radio and television communications were seen to be partially useful but it was noted that little attention was given to press, radio and television stations that are accessed by many in BME communities.

Respondents felt that little consideration appeared to be given to race or culture when the campaign was designed. Groups identified a number of steps (in addition to addressing the problems identified in this section) that could be taken to make the campaign more relevant and effective. One example was to use simpler but more positive messages that involve key opinion formers including faith leaders and health professionals. They also suggested targeting community newspapers (especially free newspapers), community radio channels and key satellite television channels that use a range of languages. Perhaps most importantly it was argued that the use of the word 'me' in 'see me' should be changed to something less individualistic, and that the slogan 'one in four' was inappropriate. New media was seen to be entirely appropriate by younger generations.

Overall, discussions centred on the value of community involvement from the outset in order to have relevant campaigns that reach those who need it most. Within communities, generations have very different views and a series of mini campaigns were suggested in addition to modifications to the existing national approach. However, respondents were generally pessimistic about the likelihood of an informationgiving campaign being successful in addressing deeply held prejudices. 


\section{New approaches}

When asked more freely about what approaches would work to address mental health stigma within their communities, a very different set of priorities and actions emerged challenging classic social marketing and public education models. A set of linked solutions and activities were suggested which are characterised by some core processes; community development, personal contact, dialogue and respecting different beliefs. These approaches were felt to be more likely to address some of the fundamental stigma that people with mental illness faced, specifically being seen as 'dangerous', to 'blame', and 'unsuitable for marriage'. Three approaches were favoured.

Participants stressed the embedded nature of stigma within communities, and the different beliefs that people held about what mental illness is, what causes it, and its impact. It was consistently stated that conversation and dialogue would be much more effective than just receiving information in writing or adverts. Across the groups it was felt that this form of community dialogue or workshops would be culturally appropriate, and more effective if delivered by people from within the community. This finding is not new and has been found in many other studies across communities who are disempowered for a range of health issues. Back in 1988 Wallerstein and Bernstein (Wallerstein \& Bernstein, 1988) critiqued how health promotion has persistently used community-wide programmes that have targeted individual behaviours and have failed to reach poor and minority sections of communities. Countering this model with ideas from Freire they argued knowledge does not come from 'experts inculcating their information and imposing their cultural values, but collective knowledge should emerge from group-sharing experiences, where people can engage in mutual reflection and authentic dialogue'. This model, advocated by communities, would allow people to explore their views and understand the impact of stigma on others. Schools, community centres, religious settings and some workplaces were seen as realistic locations from which to access a wide range of people. This approach seemed to be more popular amongst female participants in the study.

An approach that emerged very strongly amongst groups with Pakistani and Indian heritage was the potential of cultural engagement; using events and different art forms to explore mental illness and stigma. Participants highlighted how stories are often used within families and communities. They also pointed out the potential of populist cultural forms, such as Hindi cinema and drama, to engage people. Ideas ranged from developing arts programmes to influence communities, through to using preexisting, large-scale cultural events and festivals as a setting in which to engage people in a positive way.

Finally, opinion formers were viewed as very important in shaping community perceptions and responses towards people with mental illness and their families. Some, such as faith leaders, were also identified as gatekeepers to accessing services. The most significant and frequently mentioned opinion formers were religious leaders, particularly amongst those from Pakistani and Indian heritage, and most strongly from Muslim participants. Employers were also seen as key opinion formers. This was particularly so for groups of people that work long hours, and the Chinese heritage groups consistently identified the restaurant trade as an example. It is important to note opposing views that were less common but strongly felt. For example several of the female participants felt that women's groups and community development workers were more important people to engage with.

\section{IMPLICATIONS}

The findings presented here raise a number of issues that have implications for mental health policy, practice and research both locally and internationally. Overall the study confirms that stigma towards people with mental illness amongst the three BME communities was significant and deeply held, and is a significant factor in the treatment gap we outlined in the review (Patel et al., 2010). It supports aspects of the critiques of community care by authors such as Chesters (2005) who argue that merely 
being in community settings does not promote humane or effective care. The social support of families and communities is required in order for people with mental illness to live fulfilling lives and stigma remains a barrier to this.

This local study illustrated the importance of understanding and addressing stigma in its socio-cultural context. Community beliefs showed significant diversity but were frequently connected to underlying social and cultural beliefs about causation. What is clear is that we cannot assume that all sections of our communities share a medical, psychiatric view of mental illness. Mental illness is an essentially contested concept (Foucault, 1988). This is reflected in the range of services and treatments that people accessed. Stakeholders should engage communities in order to understand and respond to these social and cultural influences.

In this study stigma is viewed as a more collective experience. Families are often stigmatised as a group, and this can be due to beliefs about contagion and inheritability and blame for previous sin. It acts to reinforce the sense of shame for the individual and hiding of the problems from the wider community and health services. It suggests that in engaging with cultures that have strong community and family connectedness, we should reconsider the importance of associated stigma for families, spouses and friends. Almost half a century ago Goffman (1963) highlighted the stigma that people can face if they are closely associated with someone who is labelled as having a mental illness, particularly families. Current campaigns and mental health services primarily focus upon the individual, reflecting wider political trends in affluent western societies. However, the findings suggest we should give greater attention to this dimension of stigma and challenge individualism as the only basis of health actions and campaigns.

Responses to the national campaign force us to reconsider the value of general approaches in mental health awareness campaigns and service provision. The national anti stigma campaign, in aiming to make best use if its resources, favoured a single approach. This arguably aimed to provide the greatest health gain at a population level within its resources. The risk though is that it fails to reach those who experience multiple disadvantage, thus widening mental health inequalities, and worsening the experiences of stigma by those at most risk. This consequence appears to be borne out across the three communities. The implication is that we need to rebalance campaigns and develop them with communities, recognising the diversity between and within them. The CBPR model adopted in this study means that key organisations can use these findings to collectively address stigma.

Whilst these findings relate to three BME communities in Scotland specifically, they do raise questions for policy makers more generally about the value of persisting with public education approaches as a route to tackling stigma at all. Anti stigma campaigns are rooted in positivistic notions of self and society. Campaigns are frequently dominated by medical models and health education messages. This frames the problem as ignorance of 'facts' and sees the challenge as providing the 'right' information to those who are less well informed in the 'right way'. The public will then rationally modify the way that they interact with people with mental illness. Seen in this context, its sociological naivety is clear. Just as it failed to engage at all with communities holding diverse beliefs, it ignores the barriers to change such as prejudice, and power relationships. There are interesting links between the community-generated preferences for tackling stigma in this study and wider evidence about 'what works'. There is evidence that positive contact, narratives and the arts can be effective in reducing stigma (Corrigan, River, Lundin, \& Penn, 2001; Knifton et al., 2010). So an over-reliance on education campaigns perhaps partially explains the persistence of stigma more widely.

This study provides important insights into some of the social and cultural issues in three BME communities which affect how they respond to mental illness and mental health services. It provides insights into why stigma 
persists and why existing approaches to tackling stigma are ineffective. Our respondents were typical rather then representative meaning themes that emerged are tentative and hypothesis generating. However, an emerging potential model for national campaigns must place greater emphasis upon community development, positive contact, community dialogue and engaging with diverse beliefs. Such approaches need to understand and respond to the socio-cultural needs of communities. To succeed, mental health campaigns must develop more effective partnership with community agencies, or risk magnifying existing inequalities.

\section{REFERENCES}

Arthur, B., Knifton, L., Park, M., \& Doherty, E. (2008). Cutting the dash: Experiences of mental health and employment. Journal of Public Mental Health, 7(4), 51-59.

Berzins, K. M., Petch, A., \& Atkinson, J. M. (2003). Prevalence and experience of harassment of people with mental health problems living in the community. British Journal of Psychiatry, 183(12), 526-533.

Blackwell, M. J. (1997). Chinese mental health issues in Britain. London, England: Chinese Mental Health Association/Mental Health Foundation.

Braunholtz, S., Davidson, S., Myant, K., \& O'Connor, R. (2006). Well? What do you think? The third National Scottish Survey of public attitudes to mental health, mental wellbeing and mental health problems. Edinburgh, Scotland: Scottish Government.

Bryman, A. (2004). Social research methods. Oxford, NY: Oxford University Press.

Cargo, M., \& Mercer, S. L. (2008). The value and challenges of participatory research: Strengthening its practice. Annual Review of Public Health, 29, 325-350.

Chakraborty, A. T., McKenzie, K. J., Hajat, S., \& Stansfeld, S. A. (2010). Racism, mental illness and social support in the UK. Social Psychiatry and Psychiatric Epidemiology, 45(12), 1115-1124.

Chesters, J. (2005). Deinstitutionalisation: An unrealised desire. Health Sociology Review, 14(3), 272-282.

Corbin, J., \& Strauss, A. (2008). Basics of qualitative research:Techniques and procedures for developing grounded theory. Thousand Oaks, CA: Sage.

Corrigan, P. W., River, L. P., Lundin, R. K., \& Penn, D. L. (2001). Three strategies for changing attributions about severe mental illness. Schizophrenia Bulletin, 27(2), 187-195.

Crisp, A., Gelder, M., Rix, S., Meltzer, H., \& Rowlands, O. (2000). Stigmatisation of people with mental illnesses. British Journal of Psychiatry, $177,4-7$.

Fabrega, H. (1991). Psychiatric stigma in non-Western societies. Comprehensive Psychiatry, 32(6), 534-551.

Fabrega, H. (1992). The role of culture in a theory of psychiatric illness. Social Science and Medicine, 35(1), 91-103.

Faulkner, A., \& Layzell, S. (2000). Strategies for living. London, England: Mental Health Foundation.

Fernando, S. (2003). Cultural diversity, mental health and psychiatry. The struggle against racism. Hove, England: Brunner-Routledge.

Foucault, M. (1988). An aesthetics of existence. Sheridan, A., trans. In L. D. Kritzman (Ed.), Politics philosophy culture. Interviews and Other Writings 19771984. London, England: Routledge.

Friedli, L. (2009). Mental health, resilience and inequalities. Copenhagen, Denmark: World Health Organization and Mental Health Foundation.

Gary, F. (2005). Stigma: Barrier to mental health care amongst ethnic minorities. Issues in Mental Health Nursing, 26(10), 979-999.

Glaser, B., \& Strauss, A. (1967). The discovery of grounded theory: Strategies for qualitative research. Chicago, IL: Aldine Publishing Company.

Goffman, E. (1963). Stigma: Notes on management of spoiled identity. Upper Saddle River, NJ: Prentice Hall.

Grant, S., \& Jackson, J. (2005). Equal services. Edinburgh, Scotland: National Resource Centre for Ethnic Minority Health.

Hatfield, B., Mohammad, H., Ahim, Z., \& Tanweer, H. (1996). Mental health and the Asian communities: A local survey. British Journal of Social Work, 26(3), 315-336.

Heim, D., \& MacAskill, S. (2006). Black and minority ethnic health in greater Glasgow. Glasgow, Scotland: Greater Glasgow NHS.

Israel, B. A., Schulz, A. J., Parker, E. A., \& Becker, A. B. (1998). Review of communitybased research: Assessing partnership approaches to improve public health. Annual Review of Public Health, 19, 173-202.

Kleinman, A. (1987). Anthropology and psychiatry: The role of culture in cross-cultural research on illness. British Journal Of Psychiatry, 151(4), 447-454.

Knifton, L., \& Quinn, N. (2008). Media, mental health and discrimination: A frame of reference for 
understanding reporting trends. International Journal of Mental Health Promotion, 10(1), 23-31.

Knifton, L., Gervais, M., Newbigging, K., Mirza, N., Quinn, N., Wilson, N., \& Hunkins-Hutchison, E. (2010). Community conversation: Addressing mental health stigma with ethnic minority communities. Social Psychiatry and Psychiatric Epidemiology, 45(4), 497-504.

Lauber, C., \& Rossler, W. (2007). Stigma towards people with mental illness in developing countries in Asia. International Review of Psychiatry, 19(2), 157-178.

Li, P. L., Logan, S., Yee, L., \& Ng, S. (1999). Barriers to meeting the mental health needs of the Chinese community. Journal Public Health Medicine, 21(1), 74-80.

Link, B. G., \& Phelan, J. C. (2001). Conceptualising stigma. Annual Review of Sociology, 27(1), 363-385.

Link, B. G., \& Phelan, J. C. (2006). Stigma and its public health implications. The Lancet, 367(9509), 528-529.

Maclean, U. (1969). Community attitudes to mental illness in Edinburgh. British Journal of Preventative and Social Medicine, 23(1), 45-52.

Marmot. (2010). Marmot review - Fair society, healthy lives: Strategic review of health inequalities in England post-2010. London, England: Marmot Review.

Minkler, M., \& Wallerstein, N. (2003). Introduction to community-based participatory research. In $\mathrm{M}$. Minkler \& N. Wallerstein (Eds.), Community-based participatory research for health. San Francisco, CA: Jossey-Bass.

Minkler, M. (2004). Ethical challenges for the 'outside' researcher in community based participatory research. Health Education and Behaviour, 31(6), 684-697.

ED4 Myers, F., McCollam, A., \& Woodhouse, A. (2005). Equal minds: Addressing mental health inequalities in Scotland. Edinburgh, Scotland: Scottish Executive.

$\mathrm{Ng}$, C. H. (1997). The stigma of mental illness in Asian cultures. Australian and New Zealand Journal of Psychiatry, 31(3), 382-390.

Patel, V., Maj, M., Flisher, A. J., De Silva, M. J., Koschorke, M., Prince, M., \& WPA Zonal and Member Society Representatives. (2010). Reducing the treatment gap for mental disorders: A WPA survey. World Psychiatry, 9(3), 169-176.

Pickett, K. E., James, O. W., \& Wilkinson, R. G. (2006). Income inequality and the prevalence of mental illness: A preliminary international analysis.
Journal of Epidemiology and Community Health, 60(7), 646-647.

Rogers, A., \& Pilgrim, D. (2003). Inequalities and mental health. London, England: Palgrave Macmillan.

Rosenfield, S. (1997). Labelling mental illness: The effects of received services and perceived stigma on life satisfaction. American Journal of Sociology, 62(4), 660-672.

Schomerus, G., \& Angermeyer, M. C. (2008). Stigma and its impact on help-seeking for mental disorders: What do we know? Epidemiologica Psichiatria Sociale, 17(1), 31-37.

Silverman, D. (2004). Doing qualitative research (2nd ed.). London, England: Sage.

Tabassum, R., Macaskill, A., \& Ahmad, I. (2000). Attitudes towards mental health in an urban Pakistani community in the United Kingdom. International Journal of Social Psychiatry, 46(3), 170-181.

Van Brakel, W. (2006). Measuring health-related stigma: A literature review. Psychology, Health and Medicine, 11(3), 307-334.

Wallerstein, N., \& Bernstein, E. (1988). Empowerment education: Freire's ideas adapted to health education. Health Education and Behaviour, 15(4), 379-396.

Wenger, E. (1998). Communities of practice: Learning, meaning and identity. New York, NY: Cambridge University Press.

Williams, R., \& Hunt, K. (1997). Psychological distress among British South Asians: The contribution of stressful situations and sub-cultural differences in the West of Scotland Twenty-07 study. Psychological Medicine, 27(5), 1173-1181.

Williams, B., Copestake, P., Eversley, J., \& Stafford, B. (2008). Experiences and expectations of disabled people. London, England: Office for Disability Issues.

Wong, L., \& Richman, J. (2004). Chinese understanding of diankuang in a metropolitan city in the United Kingdom. International Journal of Mental Health, 32(3), 5-30.

World Health Organization. (2001). The world health report 2001 - Mental health: New understanding, new hope. WHO: Geneva, Switzerland.

Wright, E. R., Gronfein, W. P., \& Owens, T. J. (2000). Deinstitutionalization, social rejection, and the self-esteem of former mental patients. Journal of Health and Social Behaviour, 41(1), 68-90.

Yeung, E. (2004). Improving accessibility to mental health services for Chinese people. Liverpool, UK: Merseyside Health Action Zone.

Received $x x$ Month 20xx Accepted xx Month 20xx 


\section{Queries}

ED1 Please confirm whether the inclusion of short running title 'Understanding and addressing the stigma of mental illness' is fine.

ED2 Please provide Acknowledgements details.

ED3 Please check the inclusion of Chapter title in the reference 'Foucault, 1988'.

ED4 Please check the editing made to the reference 'Myers et al., 2005'.

ED5 Please provide recieved and accepted date details. 